Canadian

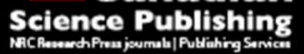

Canadian Journal of Civil Engineering Revue canadienne de génie civil

\title{
Minimizing the torsional response of inelastic multistory buildings with simple eccentricity
}

\begin{tabular}{|r|l|}
\hline Journal: & Canadian Journal of Civil Engineering \\
\hline Manuscript ID & cjce-2015-0091.R1 \\
\hline Manuscript Type: & Technical Note \\
\hline Date Submitted by the Author: & 08-Jul-2015 \\
\hline Complete List of Authors: & $\begin{array}{l}\text { Georgoussis, George; School of Pedagogical and Technological Education } \\
\text { (ASPETE), Department of Civil and Construction Engineering }\end{array}$ \\
\hline Keyword: & $\begin{array}{l}\text { response-earthquake load < Struct.Eng. \& Constr.Mate, structural } \\
\text { engineering < Computer Applications }\end{array}$ \\
\hline & \multicolumn{2}{|l}{} \\
\hline
\end{tabular}

SCHOLARONE ${ }^{\mathrm{m}}$

Manuscripts 
Minimizing the torsional response of inelastic multistory buildings with simple eccentricity

George K. Georgoussis*

* Professor, Department of Civil and Construction Engineering, School of Pedagogical and Technological Education (ASPETE), N. Heraklion 14121, Attica, Greece, e-mail: ggeorgo@tee.gr 


\begin{abstract}
Common types of multistory buildings, detailed according to a planar static analysis under a code lateral loading, may have a practically translational behavior when the mass axis is passing through (or within a close distance from) the modal center of rigidity (m-CR). The concept of this point in elastic multistory structures was introduced by the author in earlier papers, but it retains its value in structures composed by inelastic bents, since it specifies the optimum arrangement of these structural elements in terms of producing minimum torsional response in the case of a strong ground motion. This is demonstrated in typical five story buildings, composed by moment resisting frames and structural walls, under the ground motions of Kern County and Loma Prieta.
\end{abstract}

Key words: earthquake engineering, inelastic structures, strength eccentricity, modal center of rigidity 


\section{Introduction}

The rotational response of building structures has proved to be a serious cause of severe damage during strong earthquakes. In elastic single-story buildings with rigid floor diaphragms, this response is due to the eccentricity of the centre of rigidity $(\mathrm{CR})$ from the centre of mass $(\mathrm{CM})$. The response of such eccentric systems has been extensively analyzed in the past (e.g. Tso and Dempsey 1980) and an optimal, simply translational response, may be obtained when a coincidence of CR with CM is achieved. Such systems, with elastoplastic elements, having a strength distribution proportional to the stiffness distribution (usually called torsionally balanced (TB) models) also present a purely translational inelastic response under strong ground excitations and for this reason they are used as 'reference' models in relevant studies (e.g. Correnza et al. 1994).

The response of multi-story structures is much more complicated from that of single-story systems. At first, in the elastic phase, there is not always a vertical axis with properties similar to those of CR in single story systems. Only in proportionate buildings (the class of buildings in which the stiffness matrices of the various bents are proportional to each other) there is a vertical axis with the aforementioned properties. This axis is usually referred as the 'elastic axis', but such buildings are rare in practice and there are a number of reasons indicating that a combination of different types of bents provides a much more efficient lateral resistance during a strong ground motion (Paulay and Priestley 1992). Note here that most of the building codes specify the torsional provisions in relation to the centre of rigidity without a clear definition about this point in the general case of a multi-story building (Tso 1990).

Reviewing the literature, it may be seen that many investigations have been concerned with the issue of establishing a set of points located at floor levels of a multi-story building with properties similar to those of CR of single-story systems. Early studies on elastic multi-story systems (e.g. 
Smith and Vezina 1985) have led to different definitions about the eccentricity of CR from CM. Cheung and Tso (1986) proposed the 'rigidity centers (CRs)' for structural applications and the need to determine a vertical axis (at least in uniform over the height building structures) which can be identified as a minimum torsion axis has led to many approximations. Makarios and Anastassiadis (1998) introduced the concept of the 'axis of optimum torsion' and an alternative mathematical procedure was proposed by Marino and Rossi (2004).

In recent years the rotational response of multistory inelastic asymmetric structures has received major attention (a recent qualitative review has been presented by De Stefano and Pintucchi 2008) and an alternative strategy for controlling this response is presented by Aziminejad et al. (2008). In this study the problem of element strength distribution on the rotational response of the structure is studied by using a proper configuration of the centers of mass, strength and stiffness according to the findings obtained from single story systems with elements having strength dependant stiffness (Myslimaj and Tso 2002), as was suggested by Paulay (2001).

The concept of the modal center of rigidity (m-CR) was introduced by the author in earlier papers, (Georgoussis 2010, 2012). This is the center of the element modal stiffnesses and for common medium height regular buildings, where the first mode of vibration is the dominate mode, it may be determined from the first mode frequencies of the component planar bents (structural walls, moment resisting frames, coupled wall systems, wall-frame assemblies) that provide the lateral resistance of a given structure. It has been shown that in elastic mixed-bent-type multi-story building structures, regular in height, in which the vertical line of the centers of mass at the floor levels (mass axis) is practically passing through $\mathrm{m}-\mathrm{CR}$, minimum torsion is developed in the case of a ground excitation. In other words, a structural arrangement which provides a location of mCR close to the mass axis represents a structural model of minimum torsional response or a model that it is basically responding in a translational mode. In such cases, the effective seismic forces 
developed on a medium or low height structure are basically proportional to the first translational mode of vibration. Therefore, a strength assignment obtained from a planar static analysis under a set of lateral loads simulating the aforesaid mode of vibration, represents a system in which all potential plastic hinges at the critical sections (at the ends of the beams and the foot of the ground floor columns and walls, according to the strong column-weak beam concept) are formed at the same instant. This static analysis can be further simplified by assuming that the lateral design load has the shape of the code 'seismic loading' (e.g. the shape of inverted triangle) and the moments developed at the critical sections may be taken as the yield moments at the potential plastic hinges. Here it should be mentioned that to apply the aforesaid methodology in real structures is not an easy task. Not only because the locations and the size of the lateral load resisting elements are practically predetermined by architectural or aesthetic considerations, but also because other aspects should be taken into account, as for example the presence and the magnitude of the gravity loads, which add complexity to the problem.

The aim of the present work is to demonstrate that inelastic multi-story systems, uniform over the height with the mass axis practically passing through m-CR, constitute torsionally balanced systems when the element strength assignment is obtained from a planar static analysis under a set of lateral forces simulating a 'seismic loading'. That is, the response of these systems is basically translational not only in the elastic phase, but also in the inelastic region. This is demonstrated in 5-story building models, composed of different types of resisting bents: walls and moment resisting frames. In the models examined, inelasticity in frames was assumed to occur by allowing plastic hinges at the ends of the beams and at the foot of the ground floor columns, while in the structural walls, inelasticity was taken into account by means of plastic hinges at their bases. The initial stiffness of the various elements was considered independent of the assigned strength, as recommended by current building codes. Undoubtedly, in reinforced concrete structures the 
element stiffness is strength dependant (Paulay 2001; Priestley et al. 2007) and this implies that the application of the proposed concept in real structures is limited. This issue, however, is beyond the scope of the present work. Two characteristic ground motions (Kern County 1952-Taft, S69E and Loma Prieta 1989-Corralitos, 090), selected from the strong ground motion database of the Pacific Earthquake Engineering Research (PEER) Center (hppt://peer.berkely.edu) and scaled to a $\mathrm{PGA}=0.5 \mathrm{~g}$, are used to compare rotations and base torques of the models examined.

\section{Proposed equivalent multi-story torsionally balanced models}

To construct an inelastic multi-story torsionally balanced system, the first requirement is to have an elastic response that is practically translational under a unidirectional ground excitation. In uniform systems, composed of different types of resisting bents, this can be achieved with remarkable accuracy when the vertical mass axis practically passes through $\mathrm{m}-\mathrm{CR}$. Consider for example the monosymmetric multi-story building in Fig. 1(a). It has an orthogonal floor plan and all resisting bents are aligned along the principal axes $\mathrm{x}$ and $\mathrm{y}$ which have the origin at the center of mass of the floor plan. Because of the symmetry in x-direction, m-CR lies on the horizontal principal axis, and its $\mathrm{x}$-coordinate is given by the formula

$$
e=\frac{\sum\left(x_{j} \omega_{j 1}^{2}\right)}{\Sigma\left(\omega_{j 1}^{2}\right)}
$$

where $\omega_{j 1}$ is the first mode frequency of the $\mathrm{j}$-bent, calculated under the assumption that this bent carries the entire mass of the building, and $x_{j}$ is its location in the assumed reference system.

It is well known that in low and medium height building structures, the first mode of vibration has the dominant role in the case of a ground excitation. Therefore, as in the elastic phase a virtually translational response of these structures is obtained when the mass axis passes through m-CR, a strength assignment which is based on lateral loading proportional to the first translational mode 
of vibration specifies a system where plasticity begins simultaneously in all critical sections. In practice, this system can be derived from a planar static analysis, under a horizontal loading that has the shape of the first mode of vibration (or even the simpler 'code triangular' distribution) and the moments calculated at the critical sections can be taken as the yield moments of the corresponding potential plastic hinges. The numerical data that follow aim in demonstrating that in common buildings, where the strength assignment is implemented as above, any arrangement of the resisting bents which results in a location of $\mathrm{m}-\mathrm{CR}$ at a clear distance from the mass axis constitutes a model structure of increased rotational response, while, on the other hand, an optimum torsional response is obtained when the distance of $\mathrm{m}$-CR from the mass axis is practically negligible.

\section{Systems analyzed and observed torsional response}

Two types of structural configurations of a 5-story building, with an orthogonal floor plan 10x5m (Figs. 1(b) and (c)), are examined under the ground motions of Loma Prieta (1989-Corralitos, 090) and Kern County (1952-Taft, S69E), scaled to a PGA=0.5g. The total mass per floor is $50 \mathrm{kN} / \mathrm{m} / \mathrm{s}^{2}$, uniformly distributed over the floor slab, the radius of gyration about CM is $r=3.2275 \mathrm{~m}$, the story height is $3.5 \mathrm{~m}$ and the modulus of elasticity is assumed equal to $20 \times 10^{6} \mathrm{kN} / \mathrm{m}^{2}$. In both types of building models the ground motion is assumed purely translational along the y-axis and the lateral resistance along this direction is provided with three resisting elements, two of which are flexural shear walls (W1, W2) of a cross section $30 \times 220 \mathrm{~cm}$ and the other a moment resisting frame (FR) with two columns $60 \times 60 \mathrm{~cm}, 5 \mathrm{~m}$ apart, which are connected by floor beams $35 \times 50 \mathrm{~cm}$. All resisting elements (bents) are assumed to have only in-plane stiffness and their strength assignment is based on a planar static analysis under an external lateral loading with floor forces having the shape of 
the 'inverted triangle' and summing to a base (design) shear equal to $V_{d}=500 \mathrm{kN}$ (approximately equal to one fifth of the total weight of the structure). More specifically, allowing for plastic hinges at the bases of walls W1 and W2 and detailing frame FR according to the strong columnweak beam philosophy (that is, allowing plastic hinges at the ends of the beams and at the foot of the ground floor columns), this static analysis provides the following data: (i) the bending (yield) capacity at the plastic hinges at the bases of the walls equals $2233 \mathrm{kNm}$ and, (ii) the bending (yield) capacity of the plastic hinges at the ends of the beams of FR (from the top downwards) is equal to $156,195,183,158$ and $100 \mathrm{kNm}$ respectively, while the corresponding capacity at the plastic hinges at the base of the ground columns of FR equals $116 \mathrm{kNm}$.

In Model 1, the lateral resistance along the $\mathrm{x}$-axis is assumed to be provided by elements located on this axis (not shown in Fig. 1(b)). In Model 2, the lateral resistance along the X-axis is provided by a pair of structural walls (Wx) located symmetrically to the axis of symmetry at the far sides of the floor plan as shown in Fig. 1(c). These walls have a cross section $30 \times 235 \mathrm{~cm}$ and the bending capacity of the plastic hinges (allowed to be formed at the base sections) is equal to $2565 \mathrm{kNm}$, which corresponds to a lateral base shear equal to $0.8 \times 500 \mathrm{kN}$. In both models the walls $\mathrm{W} 1$ and W2 are located at fixed positions (in Model 1, W1 is located at 3m on the left of CM and W2 lies on its right at 5m and, in Model 2, W2 lies on CM and W1 at a distance 2m on the left of CM), while frame FR is assumed to take any possible position along the axis of symmetry.

The expected location of FR for minimum rotational response (by assuming the $\mathrm{x}$-coordinate of $\mathrm{m}-\mathrm{CR}$, as given by eq. [1], equal to zero) was found equal to $x=-2.12 \mathrm{~m}(\bar{x}=x / r=-0.65)$ and $x=2.12 \mathrm{~m}(\bar{x}=0.65)$ for models 1 and 2 respectively. These estimates are based on the first mode frequencies 5.793/s for walls W1, W2 and 5.621/s for frame FR, calculated under the assumption that the aforesaid bents carry the entire mass of the building. 
All analyses were performed by means of the program SAP2000-V11, using inelastic link elements at the assumed locations of plastic hinges. The moment-rotation relationships of these elements were assumed bilinear with a post-yielding stiffness ratio of the generalized loaddeformation curve, equal to $4 \%$. The nonlinear response history analyses were performed using the numerical implicit Wilson- $\theta$ time integration method, with the parameter $\theta$ taken equal to 1.4. The torsional response of the assumed models under the aforementioned excitations, for any possible location of frame FR (denoted by the normalized coordinate $\bar{x}=x / r$ ), is shown in Fig. 2 . Three response parameters, obtained by time history analyses assuming a 5\% damping ratio, are shown for both the elastic and inelastic systems: top rotations, $\theta($ rads $)$, and, also, normalized base shears and base torques. The thick lines represent the peak elastic response (top rotations: $\theta_{e}$, are shown by dashed lines, normalized base shears: $\bar{V}_{e}=V_{e} / V_{d}$ by solid lines and normalized base torques: $\bar{T}_{e}=T_{e} / r V_{d}$ by dotted lines) and the corresponding thin lines represent the peak inelastic behavior $\left(\theta_{i n}, \bar{V}_{i n}=V_{i n} / V_{d}, \bar{T}_{i n}=T_{i n} / r V_{d}\right)$. In the case of Model 1, for both the assumed ground excitations, minimum rotational elastic response $\left(\theta_{e}\right.$ and $\bar{T}_{e}$ in Figs. 2(a1) and (a2)) appears when $\bar{x}=-0.59$, which is very close to the expected value $\bar{x}=-0.65$, obtained by equating eq. [1] to zero (coincident $\mathrm{m}-\mathrm{CR}$ and $\mathrm{CM}$ ). The results of the inelastic analyses (thin lines in Figures 2(a1) and (a2)) are, in general terms, lower but similar to the elastic ones and the minimum torsional response appears for a location of FR approximately equal to $\bar{x}=-0.65$. Quite similar is the response of Model 2 (Figs. 2(b1) and (b2)). Minimum rotational response $\left(\theta_{e}\right.$ and $\left.\bar{T}_{e}\right)$ of the elastic system appears when the frame FR is located at $\bar{x}=0.56$, while, when its location is displaced a little to the right, at $\bar{x}=0.59$, the inelastic system produces a minimum torsional response (minimum values of $\theta_{\text {in }}$ and $\bar{T}_{i n}$ ). Both these locations are very close to the expected one $(\bar{x}=0.65)$. A general observation obtained from the diagrams of Fig. 2 is that the rotational 
responses, $\theta_{i n}$, of the inelastic systems are, in general, lower than those of the elastic ones and the variation of $\theta_{i n}$ and $\bar{T}_{i n}$ is smoother than that of the elastic systems. The peak base shear $\bar{V}_{i n}$ is practically constant as it is determined by a deformation profile in which all bents are well into the inelastic region in the same direction. The less sensitive variation of $\theta_{\text {in }}$ and $\bar{T}_{\text {in }}$ is attributed to the fact that yielding detunes the torsional coupling, resulting in a response that is more or less translational into the inelastic phase.

\section{Conclusions}

Common mixed-bent-type multistory building structures, composed by inelastic uniform bents, with the mass axis passing through $\mathrm{m}-\mathrm{CR}$ or in a close distance from this point, constitute torsionally balanced systems when the element strength assignment is obtained from a planar static analysis under a set of lateral forces distributed over the height according to the 'inverted triangle' rule. The response of these systems is basically translational not only in the elastic phase, but also in the inelastic region. Any other arrangement of these bents forms a structural system which is expected to undergo an increased torsional response in terms of both top rotations and base torques.

\section{Acknowledgements}

This research has been co-financed by the European Union and Greek national funds through the Operational Program "Education and Lifelong Learning" - Research Funding Program: ARCHIMEDES III. The author is grateful for this support. 


\section{References}

Aziminejad, A., Moghadam, A.S. and Tso, W.K. 2008. A new methodology for designing multi-story asymmetric buildings. The $14^{\text {th }}$ World Conference on Earthquake Engineering, 14-17 October, Beijing, China.

Cheung, V.W.-T. and Tso, W.K. 1986. Eccentricity in irregular multistory buildings. Canadian Journal of Civil Engineering, 13: 46-52.

Correnza, J.C., Hutchinnson, G.L. and Chandler, A.M. 1994. Effect of transverse load-resisting elements on inelastic earthquake response of eccentric-plan buildings. Earthquake Engineering and Structural Dynamics, 23: 75-89.

De Stefano, M. and Pintucchi, B. 2008. A review of research on seismic behaviour of irregular building structures since 2002. Bulletin of Earthquake Engineering, 6: 285-308.

Georgoussis, G.K. 2010. Modal rigidity center: its use for assessing elastic torsion in asymmetric buildings. Earthquakes and Structures, an International Journal, 1(2), 163-175.

Georgoussis, G. 2012. Seismic analysis of non-proportionate eccentric buildings. Advanced Materials Research, Vol. 450-451: 1482-1488.

Makarios, T. and Anastassiadis, K. 1998. Real and fictitious elastic axis of multi-storey buildings: theory. Structural Design of Tall Buildings, 7(1): 33-45.

Marino, E.M., and Rossi, P.P. 2004. Exact evaluation of the location of the optimum torsion axis. The Structural Design of Tall and Special Buildings, 13: 277-290.

Myslimaj, B., and Tso, W.K. 2002. A strength distribution criterion for minimizing torsional response of asymmetric wall-type systems. Earthquake Engineering and Structural Dynamics, 31: 99-120.

Paulay, T. 2001. Some design principles relevant to torsional phenomena in ductile buildings.

Journal of Earthquake Engineering, 5(3):271-308.

Pauley, T. and Priestley, M.J.N. 1992. Seismic design of reinforced and masonry buildings. Wiley Interscience.

Priestley, M.J.N., Calvi, G.M. and Kowalsky, M.J. 2007. Direct displacement based design of buildings. IUSS Press, Pavia, Italy.

Smith, B.S. and Vezina, S. 1985. Evaluation of centers of resistance in multistory building structures. Proceedings of the Institution Civil of Engineers, Part 2, 79(4): 623-635.

Tso, W.K. 1990. Static eccentricity concept for torsional moment estimations. Journal of Structural Engineering, ASCE, 116(5): 1199-1212.

Tso, W.K. and Dempsey, K.M. 1980. Seismic torsional provisions for dynamic eccentricity. Earthquake Engineering and Structural Dynamics, 8: 275-289. 


\section{FIGURE CAPTIONS}

Fig. 1. (a) Typical floor plan of a mixed-bent-type multistory building; (b),(c) Plan configurations of analyzed building models.

Fig. 2. Top rotations( $\left.\times 10^{-2} \mathrm{rads}\right)$ and normalized base shears and base torques in the analyzed models under the Kern County and Loma Prieta ground motion. 

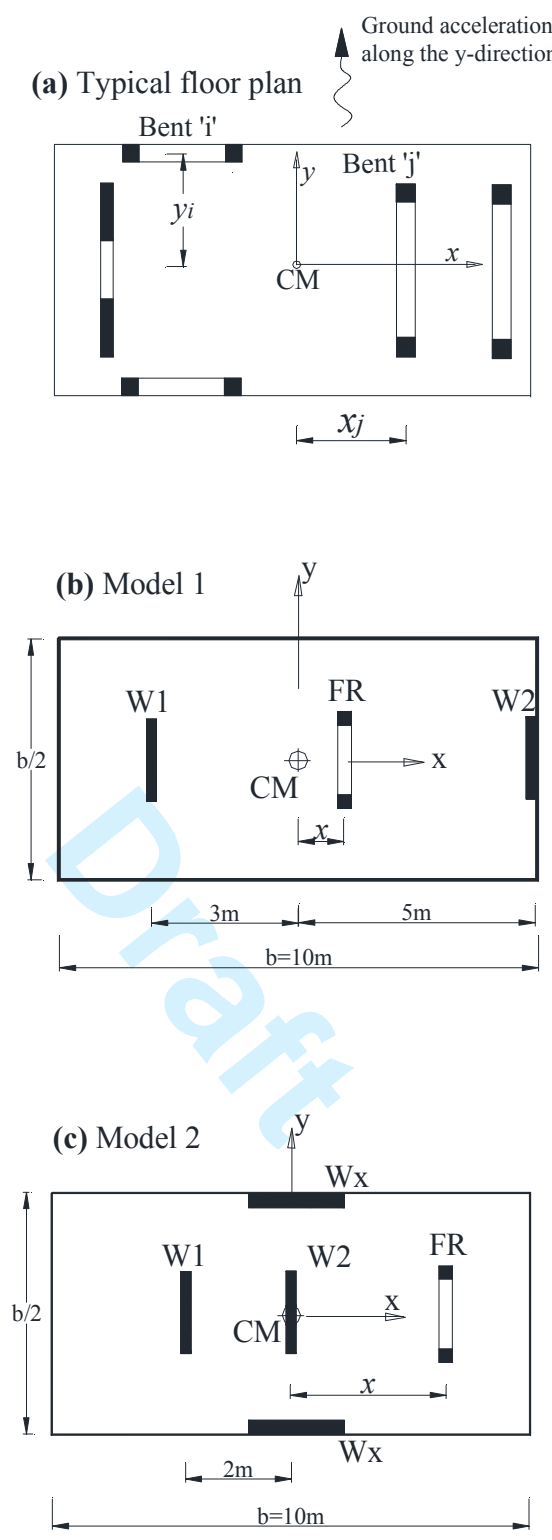

Fig. 1 

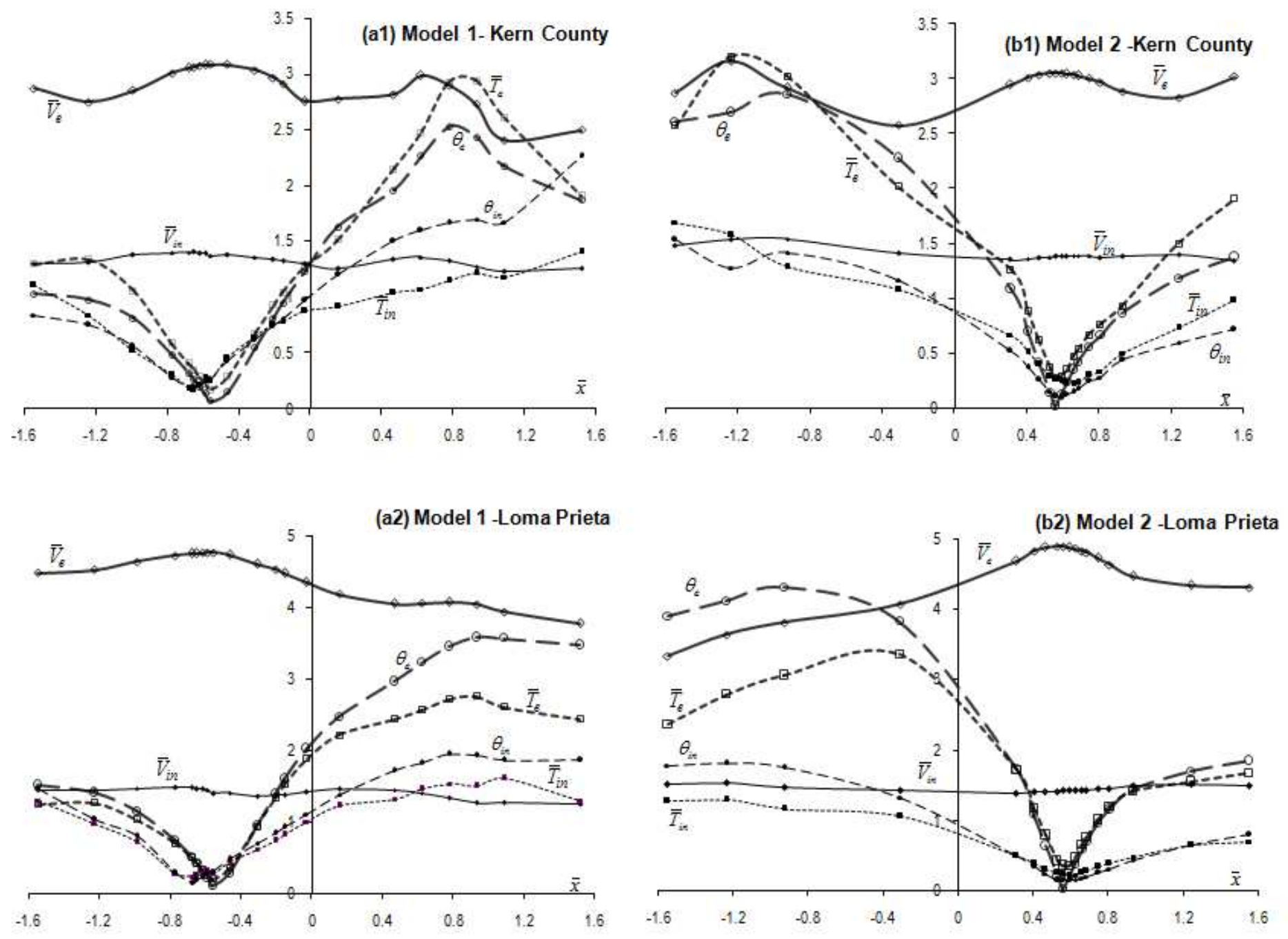

Fig. 2 